\title{
Gamma-ray absorption method (GRAM) application to the identification of EGRET unidentified sources ${ }^{\star}$
}

\author{
A. F. Iyudin ${ }^{1,2,3}$, V. Burwitz ${ }^{2}$, J. Greiner ${ }^{2}$, S. Larsson ${ }^{3}$, and A. Küpcü Yoldas ${ }^{2}$ \\ 1 Skobeltsyn Institute of Nuclear Physics, Moscow State University, Vorob'evy Gory, 119992 Moscow, Russia \\ e-mail: aiyudin@srd.sinp.msu.ru; ani@mpe.mpg.de \\ 2 Max-Planck-Institut für extraterrestrische Physik, Postfach 1312, 85741 Garching, Germany \\ 3 Stockholm Observatory, AlbaNova University Center, Roslagstullsbacken 21, 10691 Stockholm, Sweden
}

Received 7 December 2006 / Accepted 10 March 2007

ABSTRACT

\begin{abstract}
Recently we reported the very first detection of $\gamma$-ray resonant absorption along the line of sight toward $\gamma$-ray bright quasars (QSOs) like 3C 273, 3C 279, PKS 0528+0134, and BL Lacertae. These detections resulted from the analysis of absorption troughs in SEDs derived on the base of mostly EGRET data that were collected during monitoring campaigns of the Virgo and galactic anticenter regions by the Compton Gamma Ray Observatory (CGRO), as well as during ToO observations of QSOs flares.

Among three resonant absorption mechanisms that affect the $\gamma$-ray spectrum of point-like sources, we now pinpoint the $\Delta$-isobar resonance that has a very stable peak energy of the absorption cross-section for all elements (nuclei) and for individual nucleons. From two absorbers that are usually detected on the sight lines towards $\gamma$-ray bright QSOs, we concentrate here on the only one that is at the QSO rest frame.

We discuss the advantages and drawbacks of this method distinguishing between a galactic and extragalactic origin of the EGRET unidentified sources (EUIDs). This is compared to the multiwavelength identification approach that was succesfully used in a few cases to identify galactic and extragalactic objects among EUIDs. We applied the GRAM to identifying two of the EGRET unidentified sources as blazars, and predict their respective redshifts.
\end{abstract}

Key words. atomic processes - galaxies: quasars: general - gamma rays: observations

\section{Introduction}

Since the first survey of the $\gamma$-ray sky with the $\operatorname{COS} B$ satellite, the nature of most $\gamma$-ray sources has remained a mystery, as few of these sources have firmly established counterparts at other wavebands (Swanenburg et al. 1981). COS-B detected a total of 25 sources, of which only the pulsars, Crab and Vela, the molecular cloud $\rho$-Oph, and the first extragalactic source, 3C 273 were identified (Bignami \& Hermsen 1983). The remaining 21 sources in the 2nd COS-B catalog had no unambiguous counterparts at other wavebands. Following COS-B, the EGRET (Energetic Gamma-ray Experiment Telescope) onboard the Compton Gamma-Ray Observatory (CGRO) carried out improved surveys of the $\gamma$-ray sky. A total of 271 point sources of high-energy $\gamma$-rays above $100 \mathrm{MeV}$ were detected (Hartman et al. 1999). However, only a fraction of these sources were identified. The identifications include active galaxies, powered by super massive black holes, pulsars, and powerful and mysterious $\gamma$-ray bursts, which have enabled us to explore the highest energy accelerators in the cosmos.

The unidentified sources comprise 169 of the 271 sources of the $3^{\mathrm{d}}$ EGRET catalog (Hartman et al. 1999). EUIDs represent the majority of the detected $\gamma$-ray point sources, some of which in fact were originally discovered by the COS-B satellite. Resolving the mystery of the EUIDs is a challenge across all wavebands in astronomy. Among the EUIDs, two relatively bright $\gamma$-ray sources, 3EG J2016+3657, and 3EG J2020+4017, were probably detected by $\operatorname{COS} B$ under

^ Part of this work was done while AFI was visiting MPE. the names 2CG $075+00$, and $2 \mathrm{CG} 078+2$. Both these sources are projected into the heavily absorbed Cygnus region. In fact 2CG $078+2(3 E G \quad J 2020+4017)$ was thought to be a counterpart of the supernova remnant (SNR) $\gamma$-Cygni. Multiwavelength measurements were used to identify counterparts of 3EG J2016+3657 (Mukherjee et al. 2000; Halpern et al. 2001) and of 3EG J2020+4017 (Becker et al. 2004; Weisskopf et al. 2006; Bykov et al. 2004, 2006) with some success.

In this paper we explore the applicability of the gamma-ray absorption method (GRAM) to speed up and improve the identification process of EUIDs. GRAM is based on the absorption trough analysis (Iyudin et al. 2005) in the spectral energy distribution (SED). Based on the $\Delta$-resonance trough measurements of 3C 279 SED, we derived the " $\gamma$-measured" averaged redshift of 3C 279 as $z_{\mathrm{GRAM}}=0.60 \pm 0.02$ (see redshifts for 3C 279 in Table A.1). The precision of the redshift value ( $z$ ) may be as high as $\Delta z \sim 0.1$ or better, when determined using this procedure, depending on the object's $\gamma$-ray brightness and the position of the trough in the energy range of the $\gamma$-ray telescope used to produce the SED.

The application of the GRAM leads to identification of 3EG J2016+3657, and 3EG J2020+4017 with blazars at quite a high redshift of $z \approx 1.5-2.0$. These results are consistent with the results of the multiwavelength approach presented by Halpern et al. (2001) and Bykov et al. (2006). We note, though, that GRAM results can be obtained at a very modest price in time and resources, so it will be a great help to plan further identification campaigns for EUIDs and new unidentified $\gamma$-ray sources 
that are expected to be found with the forthcoming $\gamma$-ray missions AGILE and GLAST.

Unfortunately, the unidentified sources generally have large error boxes, so finding counterparts has often been a challenging job. A multiwavelength approach with X-ray, optical, and radio data is often needed for understanding the nature of these sources, but it is a very time-consuming and costly process. Many presumably dust-enshrouded high-redshift QSO are simply too faint at visible wavelengths for additional optical identification work to be done effectively. Therefore, we propose combining the multi $\lambda$ approach with the use of GRAM to speed up the identification process. Here, we demonstrate how this method (GRAM) works in the two cases of 3EG J2016+3657 and 3EG J2020+4017, i.e. two EUIDs with quite different spectra in the EGRET energy range and with different error boxes of $\theta_{95} \approx 0.55$ and of $\theta_{95} \approx 0.15$, respectively.

This paper is organized as follows. In Sect. 2 we describe an approach to the blazar identification applying multiwavelength studies. In Sect. 3 we discuss the use of GRAM for the EUIDs identification. In Sect. 4 we present our summary and directions for the future developments of the EUIDs identification. Appendix $\mathrm{A}$ is attached to demonstrate the basics of the $\gamma$-ray absorption method.

\section{EUIDs and counterpart searches}

EGRET has measured the source location, the $\gamma$-ray light curve, and the spectra of the individual $\gamma$-ray sources. Typical EGRET observations lasted for a period of about 2 weeks, although some observations were as short as a week, while others were as long as 3 to 5 weeks. EGRET's threshold sensitivity $(>100 \mathrm{MeV}$ ) for a single 2-week observation was $\sim 3 \times$ $10^{-7}$ photons $\mathrm{cm}^{-2} \mathrm{~s}^{-1}$. Details of the EGRET instrument and data analysis techniques are given elsewhere (Kanbach et al. 1988; Thompson et al. 1993a,b; Hartman et al. 1999).

It is important to point out that EGRET did not survey all regions of the sky with the same sensitivity. Because of the larger systematic uncertainties in the EGRET analysis for the highintensity Galactic plane region, the 3EG catalog (Hartman et al. 1999) adopts two different criteria for source detection thresholds. A source is listed in the catalog if it is detected at $4 \sigma$ or higher for $|b|>10^{\circ}$, and $5 \sigma$ or higher for $|b|<10^{\circ}$. Because of the differences in source sensitivities, the EGRET catalog cannot be taken as a uniform sample of the $\gamma$-ray sky, and this has to be taken into account in all source population studies.

EGRET's better sensitivity and superior angular resolution in comparison to COS-B led to nearly a ten-fold increase in the number of $\gamma$-ray source detections over COS-B. However, this did not necessarily lead to an increase in the number of source identifications. The identification of the EGRET sources, particularly those close to the Galactic plane has proved to be challenging. The error box of the typical EGRET source is large, $\sim 0.5^{\circ}-1^{\circ}$, and identifications and counterpart searches has been difficult on the basis of position alone. This is hampered further for the low-latitude sources by the presence of bright Galactic diffuse emission along the plane. Also, a lack of tight correlation between the $\gamma$-ray flux and other properties, like X-ray flux, core radio flux, etc., allows only the strongest sources to be identified on the basis of position alone.

Counterpart searches of $\gamma$-ray sources usually start with looking for "more of the same" kinds of sources. So far the identified sources fall into two major source classes: blazars and pulsars. Most of the blazar identifications are at high Galactic latitudes, where the source fields are less crowded, positions are better determined, and additional resources such as $\gamma$-ray flux variability, correlated with variability at radio or optical bands, make the identifications more confident. All the pulsars detected by EGRET are at low latitudes. It is therefore quite likely that at least a fraction of the unidentified sources at $b<10^{\circ}$ will belong to the pulsar class. In this case, a definite time signature will be needed in the $\gamma$-ray data, which was usually difficult in the case of EGRET data. Similarly, it is likely that a large fraction of the high-latitude unidentified sources, with better source positions obtained in the future with GLAST, will be associated with blazars.

An "elusive template" for possibly another class of $\gamma$-ray sources is provided by Geminga, the only radio-quiet pulsar in the EGRET data (see Caraveo et al. 1996, for a review). A Geminga-type object is characterized by faint optical emission, no radio emission, yet strong $\gamma$-ray and X-ray emission. Unlike classical radio pulsars, these sources behave as pulsars only in X-rays and $\gamma$-rays. Although Geminga is probably the nearest member of this class, it is possible that other candidates will be found in the era of GLAST. In fact, some of the fainter, mid-latitude EGRET sources (local Galactic population) were suggested to be Geminga-like pulsars (Gehrels et al. 2000).

\subsection{Blazars among the EGRET unidentified sources}

The majority of the identified EGRET sources are blazars (flatspectrum radio quasars and BL Lac objects) - the only kinds of AGN that EGRET has detected with any measure of confidence. Mattox et al. (1997) and Mattox et al. (2001) have studied the statistical issues concerning the identification of EGRET sources with blazars, and have presented the probability of associating individual sources with blazars. In the 3EG catalog Mattox et al. (2001) find that 46 EGRET sources may be confidently identified with blazars, while an additional 37 are plausibly identified with radio sources.

The blazars seen by EGRET all share several common characteristics: they are radio-loud, flat spectrum sources, with radio spectral indices $-0.6<\alpha<0.6$ (von Montigny et al. 1995). Most of the EGRET sources confidently identified with blazars are characterized by strong radio fluxes (>500 mJy) at $5 \mathrm{GHz}$. EGRET blazars have a continuum spectrum that is non-thermal and are characterized by strong variability and optical polarization. In counterpart searches of unidentified EGRET sources, the EGRET source is usually examined to see if it fits the blazar template. Here we describe multiwavelength studies of EGRET fields that have led to identifying of the EGRET source as a blazar.

\subsection{A blazar counterpart for 3EG J2016+3657}

This is an example of a low-latitude EGRET source, 3EG J2016+3657, that was identified as a blazar behind the Galactic plane, B2013+370. Although rare, it is certainly not unexpected that several of the "Galactic" unidentified sources will turn out to be blazars, given the isotropic distribution of the $\gamma$-ray blazar population.

3EG J2016+3657 was identified in a detailed study that was carried out on archival X-ray data, with follow-up optical observations of the $\gamma$-ray error box (Mukherjee et al. 2000). The identification was soon confirmed by Halpern et al. (2001), who concluded that B2013+370 was the most likely counterpart, after optical spectroscopic identifications of all the soft and hard $\mathrm{X}$-ray sources in the error circle of the EGRET source eliminated 
the other candidates. We discuss these results here in some detail to illustrate the multiwavelength "strategy" of the identification of $3 E G$ sources.

3EG J2016+3657 is one of two EGRET sources in the Cygnus region, the second being 3EG J2021+3719, that are probably associated with the unidentified COS-B source 2CG 075+00 (Pollack et al. 1985). The error circles of both 3EG J2016+3657 \& 3EG J2021+3716 are covered by archival X-ray imaging observations with ROSAT (PSPC and HRI) and $A S C A$, as well as Einstein IPC (Wilson 1980). Figure 1 shows the ROSAT soft X-ray $(0.2-2.0 \mathrm{keV})$ and HRI image of the region, along with the EGRET error boxes. The X-ray point source positions, marked in the figure, derived from the ROSAT analysis were used to search for counterparts to the X-ray sources.

Halpern et al. (2001a) used the MDM $2.4 \mathrm{~m}$ and the KPNO $2.1 \mathrm{~m}$ telescopes to obtain a complete set of optical identifications of all X-ray point sources within the error circles of the two EGRET sources. It turns out that apart from sources \# 1 and \# 3 in Fig. 1, the other sources in the EGRET fields are either cataclysmic variables (CVs) or Wolf-Rayet stars or binary $\mathrm{O}$ stars, all unlikely to be $\gamma$-ray emitters. Note that under some circumstances Wolf-Rayet binaries are expected to be significant gamma-ray emitters, e.g. Benaglia \& Romero (2003). See also a negative conclusion of Torres \& Domingo-Santamaria (2006) on the possibility to have a binary system among the EGRET unidentified sources. The two sources of interest in the field are the supernova remnant (SNR) CTB 87 (source \# 1) and the blazar-like radio source B2013+370 (source \#3). Of the two the blazar B2013+370 was suggested as the most likely candidate. The other source, CTB 87, is too weak and too far away to be the likely candidate, and was therefore rejected (see Halpern et al. 2001; Mukherjee et al. 2000, for details).

Other characteristics of B2013+370 support the identification with 3EG J2016+3657. B2013+370 has all the blazarlike characteristics of typical EGRET identifications - compact, extragalactic, non-thermal radio source, variable at optical and $\mathrm{mm}(90 \mathrm{GHz}, 142 \mathrm{GHz})$ wavelengths, with a $5 \mathrm{GHz}$ flux of $\sim 2$ Jy (Fig. 2). The spectral energy distribution (SED) of 3EG J2016+3657 (Fig. 3) is characterized by a synchrotron peak at lower energies, a Compton peak at higher energies, with most of the power output in $\gamma$-rays that confirm the blazar nature of the source. All these observations suggest that 3EG J2016+3657 fits the blazar template and that B2013+370 is the identification for the EGRET source.

\section{Identification of EUIDs by applying GRAM}

The $\gamma$-quanta absorption method (GRAM), introduced by Iyudin et al. (2005), can be used to measure the absorbing column in the cases of QSOs with known redshift. At the same time, this approach can be used to evaluate the redshift of objects with unknown identification (EUID). For this purpose a few sufficiently bright EUIDs were selected from the $3^{\mathrm{d}}$ EGRET cata$\log$ (Hartman et al. 1999) and their spectral energy distributions (SEDs) were analyzed using GRAM.

\subsection{EG J2016+3657 identification with GRAM}

Table 1 shows the derived parameters of the high-energy troughs of $3 E G$ J2016+3657. From the position of the presumably $\Delta$-isobar related absorption trough, a plausible redshift was derived to explain its shift to lower energy in the observer frame. The results of the GRAM application to the identification of EUIDs discussed in this paper are presented in Table 1.
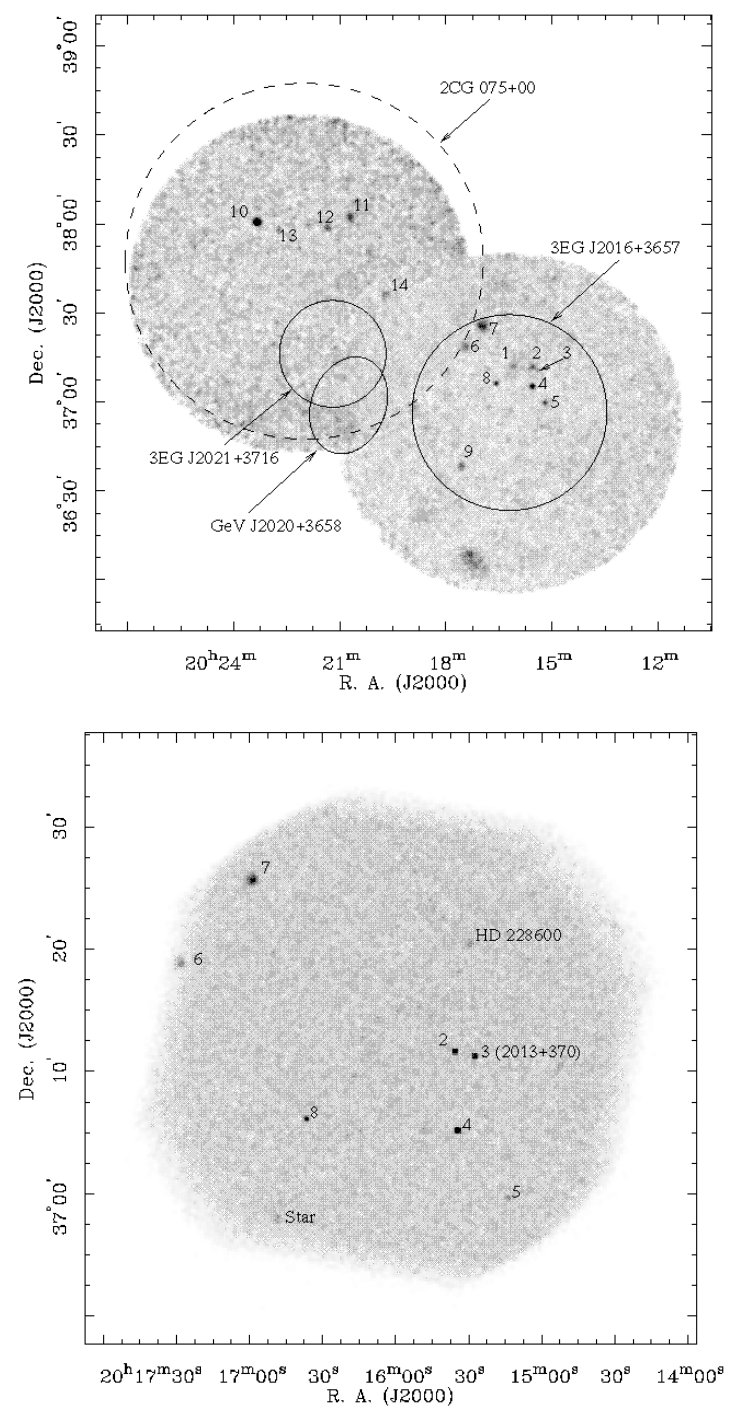

Fig. 1. (Top) ROSAT soft X-ray image of 3EG J2016+3657 and $3 E G$ J2021+3716. The circles for the two 3EG sources correspond to the $\sim 95 \%$ confidence contours. The dashed circle corresponds to the COS-B source 2CG 075+00. The GeV Catalog source (Lamb \& Macomb 1997) is also shown. The minimum detectable intrinsic flux for the ROS AT image was $6.5 \times 10^{-13} \mathrm{erg} \mathrm{cm}^{-2} \mathrm{~s}^{-1}$. (Bottom) ROSAT HRI $\mathrm{X}$-ray image of the field around 3EG J2016+3657. The image shows sources \#2 and \#3 (B2013+370) as clearly resolved point sources. Both figures are from Mukherjee et al. (2000).

Our results from fits to the SED of this source are consistent with the interpretation of the probable counterpart of 3EG J2016+ 3657 (see Halpern et al. 2001) as of a BL Lac type blazar. We found that this EUID has a redshift of $\sim 1.5-2.55$, which follows from the fit of the $\Delta$-resonance trough in SED of this source (Fig. 4, Table 1). We believe that this redshift will help for planning follow-up spectroscopic observations of B2013+3657 at this high redshift. The relative brightness of B2013+370 in the $R$ band most likely has to do with the brightness of the redshifted $\mathrm{Mg}$ II line in this galaxy and the relative weakness of the continuum. Our attempt to derive the photometric redshift of the B2013+3657 was not very successful. We have applied the publicly available software package hyperZ (Bolzonella et al. 2000) to fit the available photometry of B2013+3657. As an illustration one of the fits is shown in Fig. 5.

We have used the magnitudes (converted to fluxes) quoted by Halpern et al. (2001) at $R$ and $I$, as well as flux upper limits 
Table 1. 3EG J2016+3657 EUID, the energy of it absorption trough, and the derived redshifts.

\begin{tabular}{rccccc}
\hline \hline Object & $\chi_{\text {band }}^{2}$ & $\chi_{\text {band }+ \text { gauss }}^{2}$ & $\Delta \chi^{2}$ & $E_{1} \pm \Delta E_{1}$ & $z \pm \Delta z$ \\
\hline 3EG J2016+3657 & 46.689 & 31.858 & 14.831 & $91.53 \pm 13.25$ & $2.55 \pm 0.15$ \\
3EG J2016+3657* & 90.807 & 9.744 & 81.063 & $130.04_{-7.0}^{+6.8}$ & $1.50_{-0.13}^{+0.14}$ \\
3EG J2020+4017* & 40.132 & 8.771 & 31.361 & $131.57_{-9.0}^{+10.4}$ & $1.50 \pm 0.2$ \\
\hline
\end{tabular}

${ }^{*}$ For the case of SED with the use of the f.c.c. of EGRET.

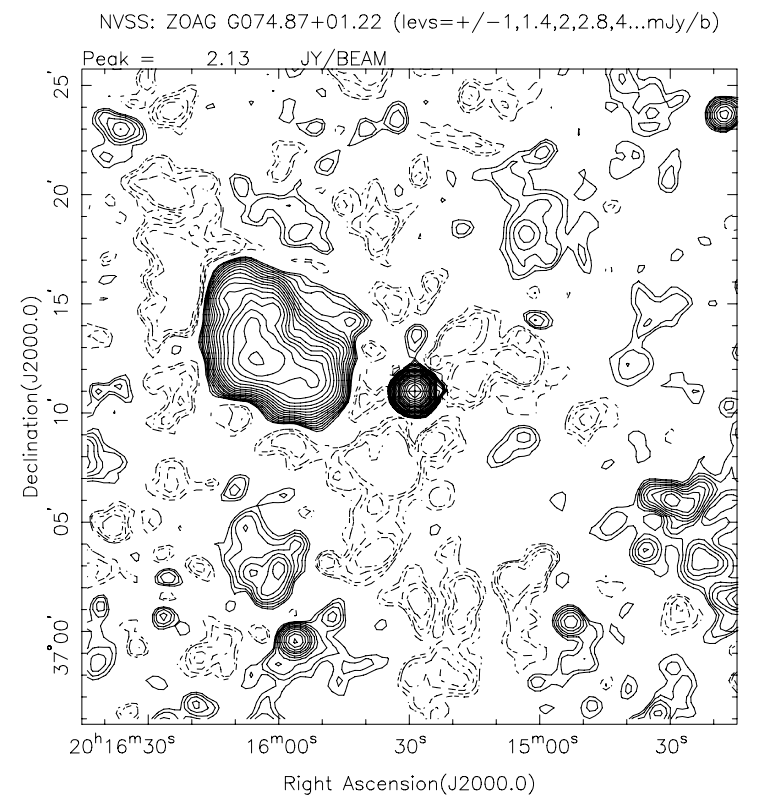

Fig. 2. NVSS image of B2013+3657 (center) shown by the contour lines. The peak intensity at the position of B2013+3657 is $2.13 \mathrm{Jy} /$ beam. Lowest contour lines are plotted at $-1,1.4,2,2.8,4 \ldots \mathrm{mJy} /$ beam.

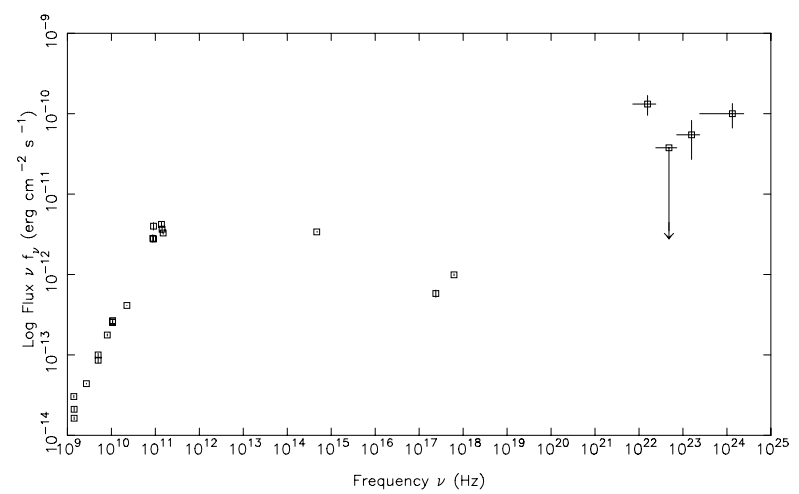

Fig. 3. Broadband spectrum of B2013+3657, assuming that its idenfitication with the $\gamma$-ray source 3EG J2016+3657 is correct. Reproduced from Mukherjee et al. (2000) to demonstrate the "blazar"-like SED of the source.

based on the limited magnitudes of 2MASS for fitting templates of different galaxy types. There are no good fits possible to the available magnitudes at filters above for the selection of redshifts from $\sim 0.1$ up to $\sim 2.0$ for all available templates of galaxy types. It is clear from the fit that the redshift is quite uncertain if it is based only on the available magnitudes at $R$ and $I$, while using lower limit of magnitudes at other filters. It will be quite interesting to compare our redshifts derived for 3EG J2016+3657 by applying GRAM with the spectroscopical redshift of B2013+3657. A side result of such a comparison will be either prove, or

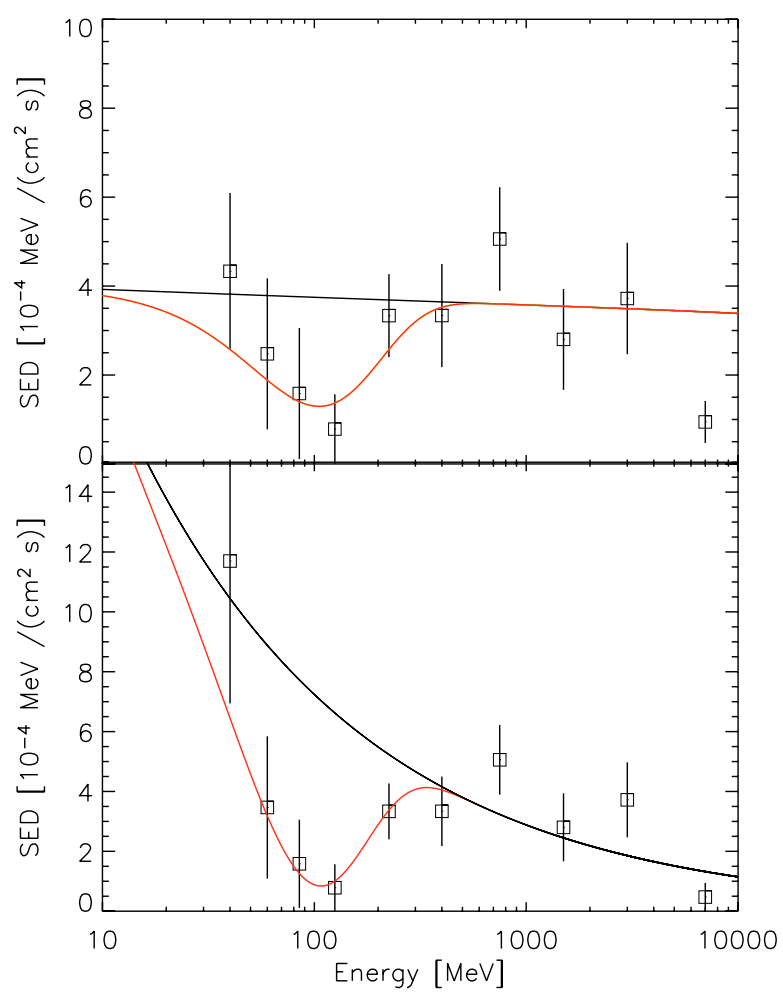

Fig. 4. Upper: a fit to the 3EG J2016+3657 SED produced without using flux correction coefficients (f.c.c.), which includes fit of the $\Delta$ resonance photon absorption in the QSO environment (red line). A black line shows the "Band"-function fit to the SED. Bottom: the same as the upper plot, but for the SED produced with the use of EGRET flux correction coefficients; see Iyudin et al. (2005) for details on the EGRET flux correction coefficients used. Boxes mark EGRET spectral points.

disprove of the necessity of using flux correction coefficients for the two lowest energy bins of EGRET SEDs. See the discussion of this point in Iyudin et al. (2005).

\subsection{EG J2020+4017: another blazar behind the Galactic plane}

3EG J2020+4017, which is also in the Cygnus region, was previously suggested as a counterpart of the SNR $\gamma$-Cyg. This suggestion led to quite intensive multiwavelength campaigns that involved X-ray observations by Chandra (Becker et al. 2004; Weisskopf et al. 2006), as well as reanalysis of the archival ROSAT data and radio observations with the Green Bank Telescope (Becker et al. 2004). Optical observations from visual through infrared wavelengths were used to identify $\sim 30 \mathrm{X}$-ray detected potential counterparts (Weisskopf et al. 2006). The conclusion drawn by Weisskopf et al. (2006) and Bykov et al. (2006) 


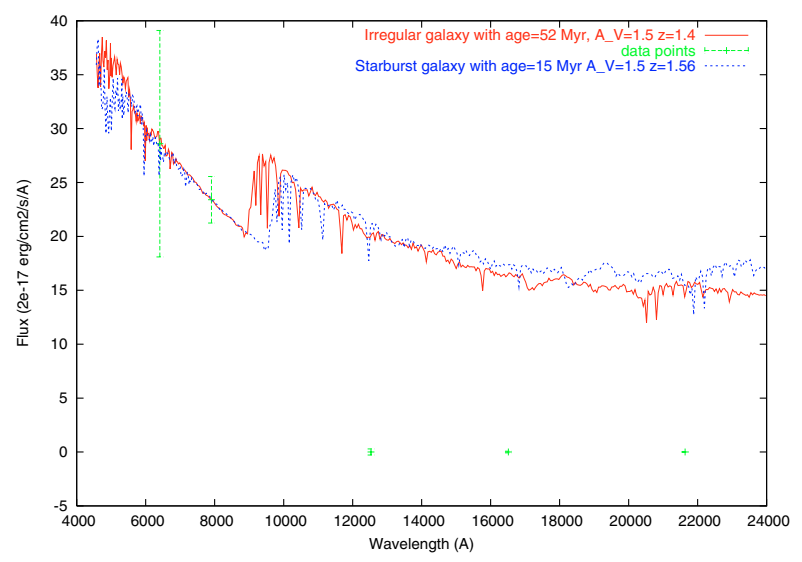

Fig. 5. HyperZ fits to templates of starburst, and irregular types for B2013+3657 magnitudes in $R$, and $I$, and of lower limits to magnitudes at $J, H$, and $K$ filters.

was that identification of a putative neutron star as a counterpart of 3EG J2020+4017 is not supported by the available data.

Bykov et al. (2006) have used INTEGRAL observations to refine positions of potential candidates for the counterpart of 3EG J2020+4017. An INTEGRAL counterpart was found in the error box of 3EG J2020+4017 as IGR J2018+4043 after observing the field with ISGRI (IBIS) (Bykov et al. 2004). Later observation of the IGR J2018+4043 with the XRT onboard Swift allowed further refining of its position, and the conclusion that it is a valid candidate for the 3EG J2020+4017 source counterpart. It was found to be quite close to the position of the galaxy 2MASX J20183871+4041003, whose magnitudes in red, $I, J, H$, and $K$ filters were derived on the base of existent data from POSSII (Reid et al. 1991) and from the 2MASS survey (Skrutskie et al. 2006). The conclusion drawn by Bykov et al. (2006) from these studies was that the most likely counterpart to the EUID 3EG J2020+4017 is indeed the 2MASX J20183871+4041003 galaxy, which has a "blazar"-like characteristics.

We verified this conclusion of Bykov et al. (2006) by applying the GRAM, i.e. via fitting the SED of 3EG J2020+4017 with a model containing the $\Delta$-isobar absorption trough. From this fit we derived the position of the trough and the redshift of the absorber that we identified with the 3EG J2020+4017 host galaxy. The redshift value we derived by applying GRAM is $z_{\text {GRAM }}=1.5 \pm 0.2$ (Table 1$)$. We also used photometric magnitudes of the 2MASX J20183871+4041003 galaxy to estimate the redshift of this object, assuming that it is a starburst galaxy. Using a starburst galaxy template is justified for this fit in view of the recently suggested overlap of the starburst activity with the QSO phase in a massive galaxy evolution, which was confirmed by observations (Carilli et al. 2001; Omont et al. 2001; Beelen et al. 2004; Page et al. 2004; Klamer et al. 2004; van Breugel et al. 2004; Staguhn et al. 2005; Roseboom et al. 2006), and is justified on theoretical grounds (Silk \& Rees 1998; Fabian 1999; Hopkins et al. 2006a,b). By again applying the hyperZ software package (Bolzonella et al. 2000), the fit gives a redshift of $z_{\mathrm{ph}}=\sim 2.0_{-0.8}^{+0.4}$. Taking errors of the photometry and of the GRAM derived redshifts into account, we find that a redshift between 1.3 and 1.7 is consistent with the results of the two different methods.

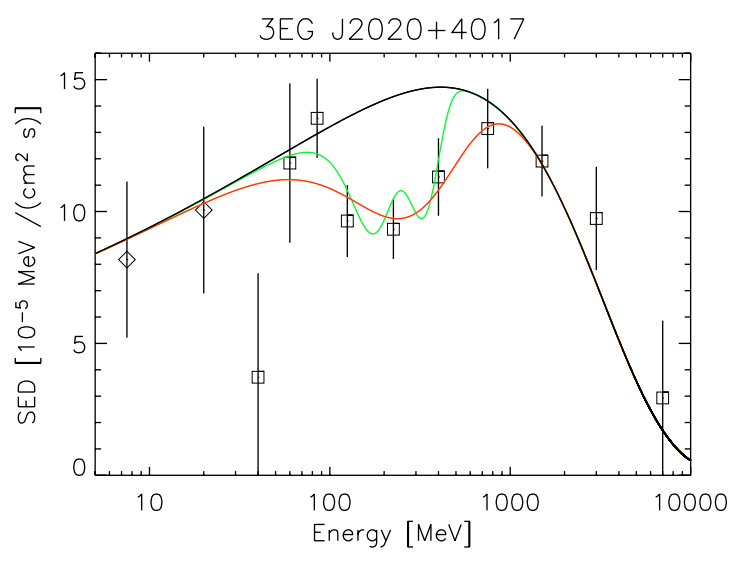

Fig. 6. Upper: a fit to the 3EG J2020+4017 SED produced with using f.c.c., which includes fits of the $\Delta$ resonance photon absorption in the QSO environment (red line). A solid green line shows the appearance of the resonance photon absorption in the host galaxy and in the local galactic environment (galactic halo) separately (not a fit). A black line shows "Band"-function fit to the SED. Diamonds show COMPTEL derived spectral points, and boxes mark EGRET spectral points.

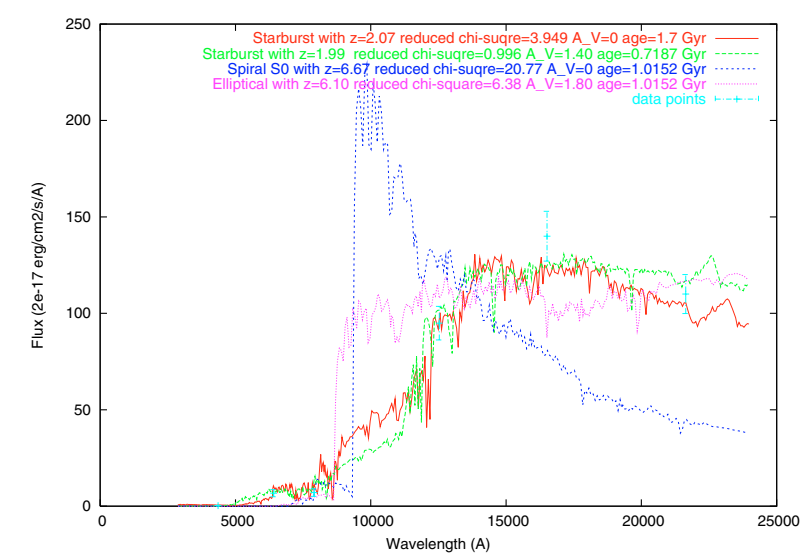

Fig. 7. HyperZ fits to templates of starburst, spiral, and elliptical types for 2MASX J20183871+4041003 magnitudes (converted to fluxes) at $B$ (lower limit), $R$, IR, $J, H$, and $K$ filters. We used magnitudes quoted by Bykov et al. (2006) to fit templates of different galaxy types. The best fit to the available magnitudes at filters above, with the reduced $\chi^{2}$ of 0.996, was found for the starburst type galaxy, with the intrinsic $A_{V}=$ 1.40 and the starburst age of $0.72 \mathrm{Gyr}$ (green line).

\section{Summary}

Resolving the mystery of the unidentified EGRET sources remains a daunting task. The strategy of identifying the EUIDs using a multiwavelength approach, although a systematic method, is a time-consuming process requiring detailed multifrequency studies of EGRET fields. It is still a successful method that has yielded several new source identifications in the past decade.

Although progress has been made in identifying individual EGRET sources, both by looking at the sources as a group and by doing follow-up multifrequency observations on a caseby-case basis, the majority of the EGRET (3EG) sources remain unidentified. Therefore, developing and applying a new and more efficient identification method is of great help for separating the EUIDs into galactic and extragalactic populations. We applied the $\gamma$-ray absorption method to the blazar identification of the $\gamma$-ray bright EUIDs in the Cygnus region, where the obscuration at optical wavelengths is very strong, and effectively 
obstructs the spectroscopical measurements of the redshift for both EUIDs for the present.

The GRAM application for identifying the EUIDs considered in this paper can be claimed as successful to the degree that the method clearly identifies both EUIDs as extragalactic objects at the significance level of $\geq 4 \sigma$. The photometrically derived redshift with hyperZ of 2 MASX J20183871+4041003, which was based on the use of the starburst template in the fitting procedure, is consistent with the redshift derived with the GRAM. In view of the clearly reddish and obscured character of the 2MASX J20183871+4041003 source, we conclude that it might belong to the class of so-called red post-starburst QSOs. They are believed to comprise between $10 \%$ and $20 \%$ of all QSOs according to different surveys (Leipski et al. 2005; Roseboom et al. 2006), which is consistent with the co-evolution of galaxy mergers, quasars, and the blue/red galaxy transitions (Hopkins et al. 2006b). We further point out that to finally derive the spectroscopical redshift of $\mathrm{B} 2013+370$, one will need an exposure on the order of $4500 \mathrm{~s}$ on the VLT class telescope equipped with a focal instrument like FORS2 to get a sufficiently good spectrum of the object with a signal-to-noise ratio of $\sim 20$.

These results clearly demonstrate the strength of GRAM in identifying extragalactic sources among the EUIDs. We believe that the GRAM will be useful for population surveys by forthcoming $\gamma$-ray missions like AGILE and GLAST. The extention of the GRAM application to cosmological studies of another type of $\gamma$-ray bright point-like sources, namely to the gammaray bursts, and especially to the long bursts with a high-energy emission component like GRB930131 or GRB980923, is rather straightforward (see Iyudin et al. 2007).

Acknowledgements. This research has made use of the NASA/IPAC Extragalactic Database (NED) operated by the Jet Propulsion Laboratory, California Institute of Technology, under contract with the National Aeronautics and Space Administration, as well as of NASA's Astrophysics Data System Service. We also acknowledge the use of the HEASARC archive. AFI acknowledges financial support from the Wenner-Gren Foundation of Sweden at the final stage of work on this paper and of the Max-Planck-Institute für extraterrestrische Physik during earlier stages of the project.

\section{Appendix A: Instruments and analysis technique}

In the following we discuss results acquired by two instruments onboard the Compton Gamma-Ray Observatory (CGRO), namely by the COMPTEL and EGRET gamma-ray telescopes, which justify the GRAM use for the EUIDs identification.

In order to determine the $\gamma$-ray spectrum of the source of interest, the EGRET energy band of $30 \mathrm{MeV}-10 \mathrm{GeV}$ was divided into 10 bins. The number of source counts in each energy interval was determined by a maximum-likelihood analysis that includes a point source (QSO or other source), a model of the diffuse background, and the presence of the other known point source(s) in the field. The diffuse emission model of Hunter et al. (1997) is generally applied. Given the diffuse background model, the source strength was determined by simultaneously optimizing the likelihood probability function with normalization of the background model and the source strength(s) of other source(s) in the field as free parameters (Mattox et al. 1996).

To verify the absence of the systematics in our method, we selected two $\gamma$-ray bright, nearby pulsars, namely Vela (distance of $\sim 400$ pc from the Sun; Cha \& Sembach 2000; Pozzo et al. 2000) and Geminga (distance of $\sim 160 \mathrm{pc}$; Caraveo et al. 1996) to analyze for the presence of absorption features in their respective SEDs. We have not found any hint of absorption in the spectra of these pulsars at the energy of most interest, i.e. $\sim 325 \mathrm{MeV}$,

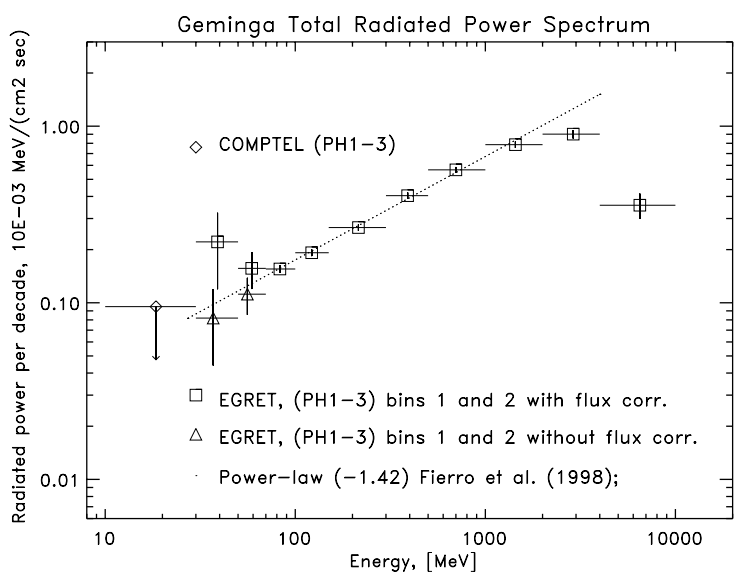

Fig. A.1. Total Geminga spectrum derived by using a spatial analysis method for EGRET data (Fierro et al. 1998) covering observations from 10 May 1991 until 04 October 1994. COMPTEL's upper limit for the 10-30 MeV energy bin shown by the diamond was taken from the paper by Kuiper et al. (1996).

the energy of the $\Delta$-resonance absorption (see Iyudin et al. 2005, and Figs. A.1, A.2).

\section{A.1. EGRET and COMPTEL spectra of QSOs}

The differential photon flux from AGNs detectable on Earth can be written as a function of the photon energy and redshift:

$\frac{\mathrm{d} N}{\mathrm{~d} E}=\left(\frac{\mathrm{d} N}{\mathrm{~d} E}\right)_{\text {unabsorbed }} \cdot \mathrm{e}^{-\tau(E, z)}$.

The dependence of $\tau$ on $E$ and $z$, however, is quite complex and cannot be cast in a simple analytical expression.

To a first order, one can assume that we are dealing with absorbers at two redshifts, one in the QSO host galaxy and the second absorber in the Milky Way. This assumption is supported by our fitting results for SEDs of the $\gamma$-ray bright QSOs (see Iyudin et al. 2005).

To produce SEDs of EUIDs (Figs. 4 and 6), and of QSOs (Figs. A.3-A.5) we have used reprocessed EGRET and COMPTEL data.

\section{A.2. Fitting procedure}

Before we proceed with the description of the fitting procedure, we note that all upper limits in the SEDs of the QSOs for the fitting purposes were written as $\operatorname{SED}_{\text {u.l. }}=1 \sigma \pm 1 \sigma$. As a first step in the fitting procedure, we fit a smooth function to the spectrum. We use the so-called "Band"-function (Band et al. 1993). While fitting this smooth function, we do not include energy bins at the suspected troughs. In this way we produce the simplest model of the specific SED. After selecting the initial fit parameters of the "Band"-function, $\alpha, \beta, E_{0}$, and $A$ (Band et al. 1993), in the second fit we use all energy bins of the SED. For this second fit we use the sum of the "Band"-function and a Gaussian as a fit function; i.e. we add another three free parameters to the fit function, namely the mean energy, the rms $(\sigma)$, and the normalization of the Gaussian. Note that the number of bins is also increased. From this fit we derive the value of $\chi_{\text {band+gauss }}^{2}$ as a quality descriptor of the SED fit by the more complex model, which is generally compared with $\chi_{\text {band }}^{2}$, i.e. a quality descriptor of the simplest model fit. The comparison of $\chi_{\text {band }}^{2}$ and $\chi_{\text {band+gauss }}^{2}$ was 


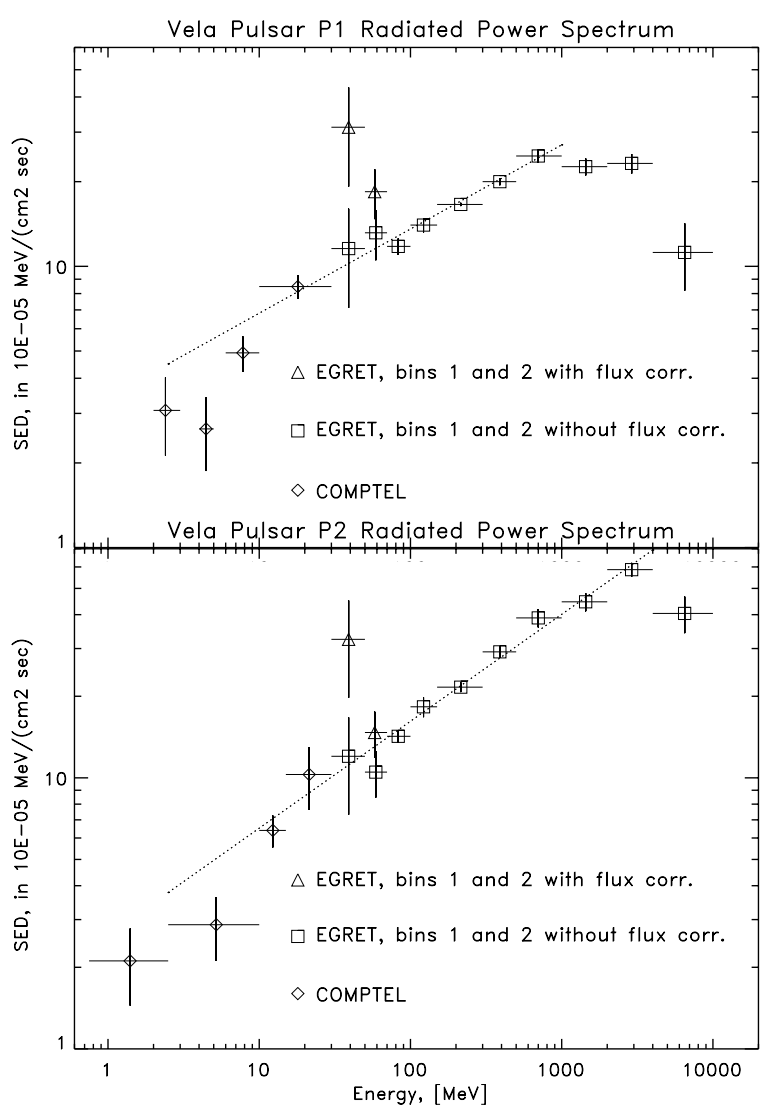

Fig. A.2. Pulsed Vela pulsar spectra for phases P1 (upper plot) and P2 (lower plot) derived by using timing analysis for COMPTEL (Kuiper et al. 1998) and EGRET data (Fierro et al. 1998), covering observations of Vela pulsar during the whole CGRO mission.

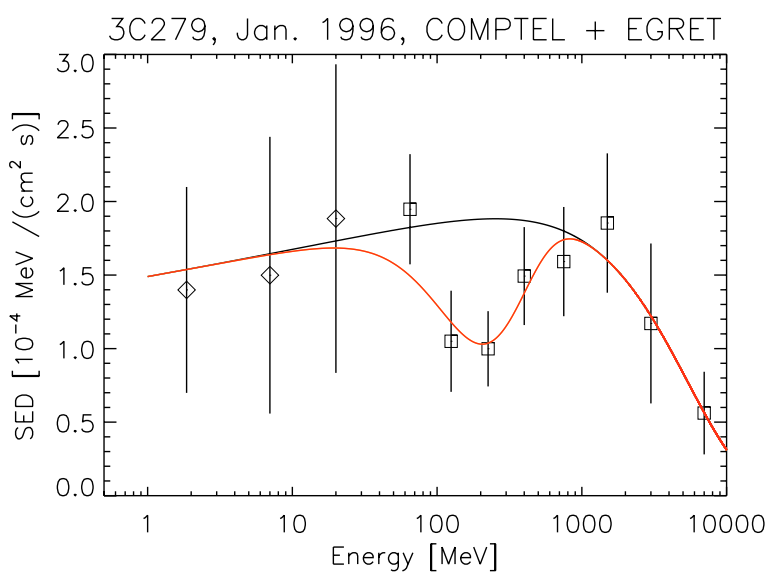

Fig. A.3. A fit to the 3C 279 spectrum measured during the flare of January 1996, which includes the $\Delta$ resonance photon absorption in the QSO circumnuclear environment. The fit improvement with the absorption line included was found to be $\Delta \chi^{2}=\chi_{\text {band }}^{2}-\chi_{\text {band }+ \text { gauss }}^{2}=36.1$ that corresponds to the probability of the line being spurious of $\sim 6 \times 10^{-8}$. Line colours, diamonds, and boxes have the same meaning as in Fig. 4.

given in Table 1 of Iyudin et al. (2005), and it shows generally very significant improvement of the fits with the absorption line included in the fit function.

Table A.1 contains values of the absorption trough position $E_{1}$ for the $\Delta$-resonance related trough of the QSO covering the redshift range from $z \sim 0.07$ for BL Lac up to $z=2.07$ for PKS0528+134. The width of these troughs is not

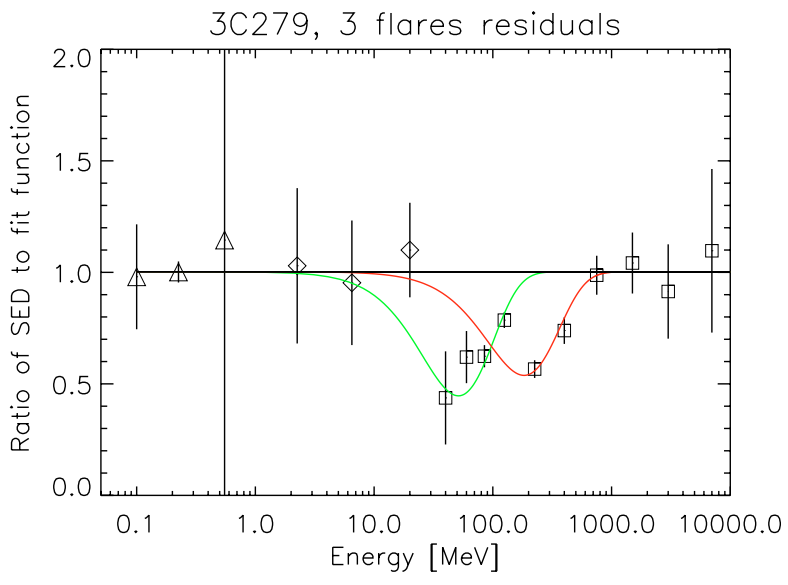

Fig. A.4. A fit to the weighted mean of the ratio of the "Band+ gauss" fit and the smooth "Band" function fit of the 3C 279 three flares SEDs (June 1991, January 1996, and February 1996). The fit contains a GDR resonance absorption feature (green line) produced in the low redshift local environment (galactic halo) and also includes the $\Delta$ resonance absorption features at the redshifts of the QSO host galaxy and of the Milky Way, as shown by the red line. The black line shows the normalised ratio of the SED to the "Band"-function fit without absorption. Diamonds and boxes have the same meaning as in Fig. 4, and triangles show OSSE points from a paper by Collmar (2003).

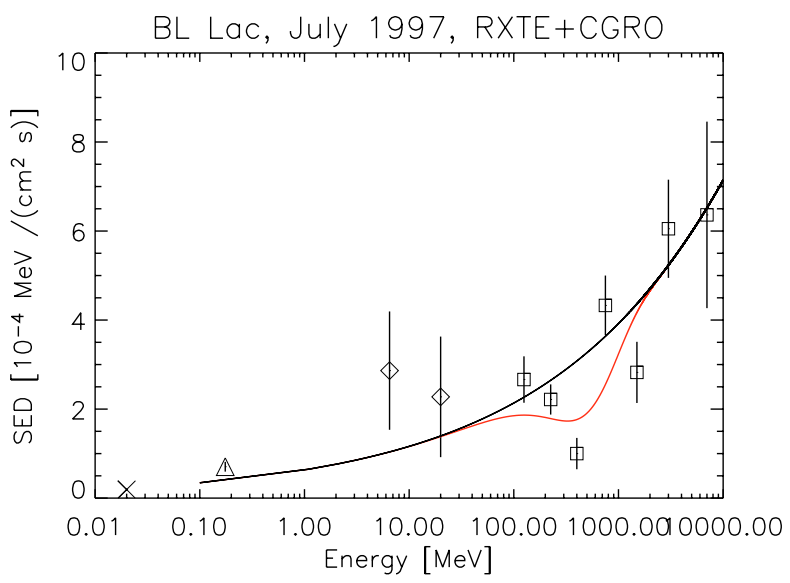

Fig. A.5. A fit to the BL Lacertae spectral energy distribution of the 1997 July flare by a fit function that includes the $\Delta$ resonance photon absorption in the circum-QSO environment (red line). The fit improvement with the absorption line included was found to be $\Delta \chi^{2}=\chi_{\text {band }^{-}}^{2}$ $\chi_{\text {band+gauss }}^{2}=23.7$, which corresponds to the probability of the line being spurious of $\sim 3 \times 10^{-5}$. The cross shows the SED fit value from the RXTE PCA measurements at $20 \mathrm{keV}$ (Madejski et al. 1999), and the triangle show the OSSE measured flux in the 50-300 keV energy interval for this flare (Grove \& Johnson 1997). Diamonds are COMPTEL measured fluxes and boxes are EGRET measurements for the BL Lac July 1997 flare. A black line is the "Band"-function fit.

included in Table A.1, but we have verified that the width of fitted $\Delta$-resonance related trough is consistent with the trough position and/or with the object redshift. In the simplest case, absorption at a particular resonance can happen at the redshift of the host galaxy, as well as at redshift zero, i.e. in the halo or disk of the Milky Way. In a more complicated case, one may observe the QSO through a number of absorbers that are having different redshifts. To decompose such a complicated absorption pattern of troughs, one would need plenty of photons and adequate energy and angular resolution of the $\gamma$-ray telescope. 
Table A.1. QSOs and their absorption lines.

\begin{tabular}{rccc}
\hline \hline Object & Observ. Time & $\begin{array}{c}E_{1} \pm \Delta E_{1} \\
\mathrm{MeV}\end{array}$ & $z \pm \Delta z$ \\
& & $225 \pm 75$ & $0.44(4) \pm 0.33(3)$ \\
3C 279 & June 1991 & $203.6 \pm 6.6$ & $0.596 \pm 0.032$ \\
3C 279 & Feb. 1996 & $207.5 \pm 24.9$ & $0.566 \pm 0.12$ \\
3C 279 & Jan. 1996 & $192.6 \pm 9.9$ & $0.687 \pm 0.051$ \\
3C 279 & Phase I-IV & $105.95 \pm 49.37$ & $2.08 \pm 0.47$ \\
PKS0528 & Mar. 1993 & $278_{-87}^{+63}$ & $0.17_{-0.22}^{+0.53}$ \\
BL Lac & July 1997 & &
\end{tabular}

\section{A.3. $3 C 279$}

The optically violent variable (OVV) quasar 3C 279, which is one of the brightest $\gamma$-ray QSOs in the EGRET energy range, was monitored by $\gamma$-ray telescopes onboard CGRO (Hartman et al. 1999). Because of its brightness it is one of the best candidates available to verify the presence of the $\Delta$-resonance absorption in the rest frame of the QSO. Figure A.3 presents the 3C 279 spectrum, simultaneously measured by COMPTEL and EGRET during the flare in January 1996. Figure A.4 shows the weighted mean ratio of the "Band+ gauss" fit and the smooth "Band" function fit for the 3C 279 flare spectra (June 1991, January 1996, and February 1996).

The $\Delta$-resonance related trough is clearly present in the SED of 3C 279 (Figs. A.3 and A.4). From the measured SEDs for this source, one can derive the weighted mean energy of the $\Delta$-resonance trough in the observer coordinate system as $E^{\text {measured }}=203.5 \pm 3.5 \mathrm{MeV}$. By using the known redshift of $3 \mathrm{C} 279$, we re-calculate the energy of the trough in the QSO rest frame as $E_{\Delta}^{\text {restframe }}=313 \pm 10 \mathrm{MeV}$. This is remarkably close to the expected value of the peak position in the absorption cross section at the $\Delta$-isobar resonance.

The flare spectra in Fig. A.4 also show evidence of another trough at energies $\sim 30 \mathrm{MeV}$, produced by giant dipole resonance (GDR) absorption. We note in passing that the GDR absorption trough shape is dependent on the metallicity of the absorbing matter (see more in Iyudin et al. 2005 on this).

\section{A.4. BL Lacertae}

BL Lacertae is another bright QSO at a redshift of $z=0.069$. BL Lac objects have been interpreted as extreme cases of a beamed source in which the Doppler boost is so large that a substantial portion of the observed optical spectrum is dominated by nonthermal emission from the jet (Blandford \& Königl 1979). BL Lac was first detected at energies $>100 \mathrm{MeV}$ by EGRET in 1995 (Catanese et al. 1997). In July 1997 during an optical flare of BL Lac, it was again significantly detected by EGRET (Bloom et al. 1997; Böttcher \& Bloom 2000) and by X-ray telescopes (Madejski et al. 1999; Grove \& Johnson 1997; Ravasio et al. 2003). During this flare the spectrum of BL Lac was significantly harder than the time-averaged spectrum of 3EG J2202+4217 (Hartman et al. 1999). This hard spectrum provided us with enough statistics to fit a $\Delta$-resonance absorption trough to the SED of July 1997. Figure A.5 shows the resulting fit, and Table A.1 provides information on the energy of the fitted trough. The redshift value of $z \sim 0.17_{-0.2}^{+0.5}$ for BL Lac, which was derived for the 1997 July flare by our absorption method, is a rather crude estimate, but it is broadly consistent with the optically measured redshift of $z=0.069$.
Detection of the $\Delta$-resonance trough in BL Lac points to the presence of a jet or of an accretion disk wind in the system during its flare state.

\section{References}

Band, D., Matteson, J., Ford, L., et al. 1993, ApJ, 413, 281

Becker, W., Weisskopf, M. C., Arzoumanian, Z., et al. 2004, ApJ, 615, 897

Beelen, A., Cox, P., Pety, J., et al. 2004, A\&A, 423, 441

Benaglia, P., \& Romero, G. E. 2003, A\&A, 399, 1121

Bignami, G. F., \& Hermsen, W. 1983, ARA\&A, 21, 67

Blandford, R. D., \& Königl, A. 1979, ApJ, 232, 34

Bloom, S. D., Bertsch, D. L., Hartman, R. C., et al. 1997, ApJ, 490, L145

Böttcher, M., \& Bloom, S. D. 2000, AJ, 119, 469

Bolzonella, M., Miralles, J.-M., \& Pello, R. 2000, A\&A, 363, 476

Bykov, A. M., Krassilchtchikov, A. M., Uvarov, Yu. A., et al. 2004, A\&A, 427, L21

Bykov, A. M., Krassiltchtikov, A. M., Uvarov, Yu. A., et al. 2006, ApJ, 649, L21

Caraveo, P., Bignami, G. F., Mignani, R., \& Taff, L. G. 1996, A\&AS, 120C, 65

Carilli, C. L., Bertoldi, F., Rupen, M. P., et al. 2001, ApJ, 555, 625

Catanese, M., Akerlof, C. W., Biller, S. D., et al. 1997, ApJ, 480, 562

Cha, A. N., \& Sembach, K. R. 2000, ApJS, 126, 399

Collmar, W. 2003, in Proc. High Energy Blazar Astronomy, ed. L. O. Takalo, \& E. Valtaoja, ASP, CP299, 29

Fabian, A. C. 1999, MNRAS, 308, L39

Fierro, J. M., Michelson, P. F., Nolan, P. L., \& Thompson, D. J. 1998, ApJ, 494, 734

Gehrels, N., Macomb, D. J., Bertsch, D. L., et al. 2000, Nature, 404, 363

Grove, J. E., Johnson, W. N., Madejski, G., et al. 1997, IAUC, 6705, 2

Halpern, J. P., et al. 2001, ApJ, 551, 101

Hartman, R. C., Bertch, D. L., Bloom, S. D., et al. 1999, ApJS, 123, 79

Hopkins, P. F., Hernquist, L., Cox, T. J., et al. 2006a, ApJS, 163, 50

Hopkins, P. F., Bundy, K., Hernquist, L., et al. 2006b [arXiv:astro-ph/0601621]

Hunter, S. D., Bertsch, D. L., Catelli, J. R., et al. 1997, ApJ, 481, 205

Iyudin, A. F., Reimer, O., Burwitz, V., Greiner, J., \& Reimer, A. 2005, A\&A, 436, 763

Iyudin, A. F., Greiner, J., Larsson, S., \& Ryde, F. 2007, in Proc. of GRB minisymposium, ed. M. Axelsson, \& F. Ryde, Stockholm September 01, 2007, AIP CP-906, in print

Kanbach, G., Bertsch, D. L., Fichtel, C. E., et al. 1988, Space Sci. Rev., 49, 69 Klamer, I. J., Ekers, R. D., Sadler, E. M., et al. 2004, ApJ, 612, L97 Kuiper, L., Hermsen, W., Bennett, K., et al. 1996, A\&AS, 120, 73

Kuiper, L., Hermsen, W., Schönfelder, V., Bennett, K., \& Connors, A. 1998, Proc. of the International Conference Many faces of neutron stars, ed. R. Buccheri, J. van Paradijs, \& M. A. Alpar (Dordrecht, Boston: Kluwer Academic Publishers), 211

Lamb, R. C., \& Macomb, D. J. 1997, ApJ, 488, 872

Leipski, C., Haas, M., Meusinger, H., et al. 2005, A\&A, 440, L5

Madejski, G. M., Sikora, M., Jaffe, T., et al. 1999, ApJ, 521, 145

Mattox, J. R., Bertsch, D. L., Chiang, J., et al. 1996, ApJ, 461, 396

Mattox, J. R., Schachter, J., Molnar, L., et al. 1997, ApJ, 481, 95

Mattox, J. R., Hartman, R. C., \& Reimer, O. 2001, ApJS, 135, 155

Mukherjee, R., Gotthelf, E. V., Halpern, J., \& Tavani, M. 2000, ApJ, 542, 740

Omont, A., Cox, P., Bertoldi, F., et al. 2001, A\&A, 374, 371

Page, M. J., Stevens, J. A., Ivison, R. J., \& Carrera, F. J. 2004, ApJ, 611, L85

Pollack, A. M. T., Bennett, K., Bignami, G. F., et al. 1985, A\&A, 146, 352

Pozzo, M., Jeffries, R. D., Naylor, T., et al. 2000, MNRAS, 313, L23

Ravasio, M., Tagliaferri, G., Ghisellini, G., et al. 2003, A\&A, 408, 479

Reid, I. N., Brewer, C., Brucato, R. J., et al. 1991, PASP, 103, 661

Roseboom, I. G., Pimbblet, K. A., Drinkwater, M. J., et al. 2006, MNRAS, 373, 349

Silk, J., \& Rees, M. J. 1998, A\&A, 331, L1

Staguhn, J. G., Stern, D., Benford, D. J., et al. 2005, ApJ, 629, 633

Strutskie, M. F., Cutri, R. M., Stiening, R., et al. 2006, AJ, 131, 1163

Swanenburg, B. N., Bennett, K., Bignami, G. F., et al. 1981, ApJ, 243, L69

Thompson, D. J., Bertsch, D. L., Fichtel, C. E., et al. 1993a, ApJS, 86, 629

Thompson, D. J., Bertsch, D. L., Dingus, B. L., et al. 1993b, ApJ, 415, L13

Torres, D. F., \& Domingo-Santamaria, E. 2006 [arXiv: astro-ph/0611360]

van Breugel, W., Fragile, C., Croft, S., et al. 2005, IAUS, 222, 485

von Montigny, C., Bertsch, D. L., Chiang, J., et al. 1995, A\&A, 299, 680

Weisskopf, M. C., Swartz, D. A., Carraminana, A., et al. 2006, ApJ, 652, 387

Wilson, A. S. 1981, ApJ, 241, L19 